Check for updates

Cite this: Phys. Chem. Chem. Phys., 2019, 21, 2480

Received 8th November 2018, Accepted 20th December 2018

DOI: $10.1039 / \mathrm{c} 8 \mathrm{cp} 06930 a$

rsc.li/pccp

\title{
Extremely large differences in DFT energies for nitrogenase models $\dagger$
}

\author{
Lili Cao (D) and Ulf Ryde (D)*
}

\begin{abstract}
Nitrogenase is the only enzyme that can cleave the triple bond in $\mathrm{N}_{2}$, making nitrogen avaiable for other organisms. It contains a complicated $\mathrm{MoFe}_{7} \mathrm{~S}_{9} \mathrm{C}$ (homocitrate) cluster in its active site. Many computational studies with density-functional theory (DFT) of the nitrogenase enzyme have been presented, but they do not show any consensus - they do not even agree where the first four protons should be added, forming the central intermediate $E_{4}$. We show that the prime reason for this is that different DFT methods give relative energies that differ by almost $600 \mathrm{~kJ} \mathrm{~mol}^{-1}$ for different protonation states. This is $4-30$ times more than what is observed for other systems. The reason for this is that in some structures, the hydrogens bind to sulfide or carbide ions as protons, whereas in other structures they bind to the metals as hydride ions, changing the oxidation state of the metals, as well as the $\mathrm{Fe}-\mathrm{C}, \mathrm{Fe}-\mathrm{S}$ and $\mathrm{Fe}-\mathrm{Fe}$ distances. The energies correlate with the amount of Hartree-Fock exchange in the method, indicating a variation in the amount of static correlation in the structures. It is currently unclear which DFT method gives the best results for nitrogenase. We show that non-hybrid DFT functionals and TPSSh give the most accurate structures of the resting active site, whereas B3LYP and PBEO give the best $\mathrm{H}_{2}$ dissociation energies. However, no DFT method indicates that a structure of $E_{4}$ with two bridging hydride ions is lowest in energy, as spectroscopic experiments indicate.
\end{abstract}

\section{Introduction}

Nitrogenase (EC 1.18/19.6.1) is the only enzyme in nature that can cleave the triple bond in $\mathrm{N}_{2}$ to form ammonia and make nitrogen available for cell metabolism. ${ }^{1-3}$ It is present in a few groups of bacteria and archaea. ${ }^{1-3}$ The nitrogenase reaction is essential to the life on earth - although $78 \%$ of the atmosphere is $\mathrm{N}_{2}$, nitrogen is often the limiting factor for plant growth and a main component of synthetic fertilisers. ${ }^{3}$ In fact, the industrial Haber-Bosch process to form ammonia consumes $\sim 1 \%$ of the world's energy supplies, produces half of the total biologically available nitrogen on earth ${ }^{4}$ and is a major factor in the recent human population explosion. ${ }^{3}$

Crystallographic studies have shown that the nitrogenase is a large $\alpha_{2} \beta_{2}$ heterotetramer. ${ }^{5-9}$ The catalytic centre is the $\mathrm{MoFe}_{7} \mathrm{~S}_{9} \mathrm{C}$ (homocitrate) FeMo cluster bound to the protein by a cysteine and a histidine residue. In some enzymes, the Mo ion is replaced by vanadium or iron. ${ }^{10}$ The protein also contains a $\mathrm{Fe}_{8} \mathrm{~S}_{7} \mathrm{Cys}_{6}$ cluster, called the $\mathrm{P}$ cluster, which transfers electrons. The electrons are provided by another protein, called the Fe protein, which binds two ATP molecules. Hydrolysis of the ATP molecules triggers the dissociation of the Fe protein, opening up for additional electron transfers.

Department of Theoretical Chemistry, Lund University, P. O. Box 124,

SE-221 00 Lund, Sweden. E-mail: Ulf.Ryde@teokem.lu.se

$\dagger$ Electronic supplementary information (ESI) available. See DOI: 10.1039/c8cp06930a
Nitrogenase catalyses the $\mathrm{N}_{2}+8 \mathrm{e}^{-}+8 \mathrm{H}^{+}+16 \mathrm{ATP} \rightarrow 2 \mathrm{NH}_{3}+$ $\mathrm{H}_{2}+16 \mathrm{ADP}+16 \mathrm{P}_{\mathrm{i}}$ reaction. It is typically described by the Lowe-Thorneley scheme, involving nine intermediates, $\mathrm{E}_{0}-\mathrm{E}_{8}$, differing in the number of electrons and protons delivered to the enzyme. ${ }^{11}$ Nitrogenase has been extensively studied by a great wealth of biochemical, spectroscopic and kinetic methods and several of the intermediates have been trapped and characterised. ${ }^{1-3,12,13}$ of particular interest is $\mathrm{E}_{4}$, which is believed to be the species that binds $\mathrm{N}_{2}$ concomitant with the release of $\mathrm{H}_{2}$ by reductive elimination. ${ }^{3}$

The experimental studies have been supplemented by many density functional theory (DFT) studies, which can give an detailed atomistic and energetic picture of the reaction mechanism. ${ }^{3,13-26}$ Unfortunately, they have led to strongly diverging mechanistic suggestions. For example, some studies have proposed that $\mathrm{N}_{2}$ is sequentially protonated first on one $\mathrm{N}$ atom, which dissociates into $\mathrm{NH}_{3}$ before the second atom is protonated, ${ }^{23,25}$ whereas other studies have suggested that it is alternatively protonated on the two $\mathrm{N}$ atoms, forming $\mathrm{N}_{2} \mathrm{H}_{2}$ and $\mathrm{N}_{2} \mathrm{H}_{4}$ intermediates. ${ }^{22,26}$ Likewise, there is no agreement on how $\mathrm{N}_{2}$ binds to the cluster, e.g. side-on to one $\mathrm{Fe}$ ion, ${ }^{22}$ with one $\mathrm{N}$ atom bridging two $\mathrm{Fe}$ ions, after the dissociation of one of the sulfide ions, ${ }^{23}$ in the centre of the cluster, displacing a triply protonated carbide ion, ${ }^{24}$ or forming a covalent bond to the carbide ion. ${ }^{25,26}$

In fact, there is not even any consensus regarding the structure of the central intermediate $\mathrm{E}_{4}$. For example, Hoffman and 
coworkers have proposed models with two protonated sulfide ions and two bridging hydride ions, ${ }^{27}$ in agreement with spectroscopic results, whereas Siegbahn has argued that it is energetically much more favourable to triply protonate the central carbide ion. ${ }^{28}$ Recently, we have performed a systematic combined quantum mechanical and molecular mechanical (QM/MM) study of the possible protonation states of the FeMo cluster, providing a great wealth of possible structures for the various intermediates in the reaction mechanism. ${ }^{29}$ Here, we examine ten of these and show extreme energy differences between different DFT methods, partly explaining the discrepancy found in previous studies. We also discuss which DFT method gives the more accurate results, by studying the geometry of the resting $\mathrm{E}_{0}$ state and $\mathrm{H}_{2}$ dissociation from $\mathrm{E}_{4}$ and $\mathrm{E}_{2}$.

\section{Methods}

\section{The protein}

All calculations are based on the $1.0 \AA$ crystal structure of nitrogenase from Azotobacter vinelandii (PDB code 3U7Q). ${ }^{7}$ The setup of the protein is identical to that of our previous studies. $^{29-31}$ The entire heterotetramer was included in the calculations and the DFT calculations were concentrated on the FeMo cluster in the $\mathrm{C}$ subunit. The $\mathrm{P}$ clusters and the FeMo cluster in subunit A were modelled by MM in the fully reduced and resting states, respectively. ${ }^{30}$

The protonation states of all residues were the same as before: ${ }^{30}$ all Arg, Lys, Asp, and Glu residues were charged, except Glu-153, 440, and 231D (a letter "D" after the residue number indicates that it belongs to that subunit; if no letter is given, it belongs to subunit $\mathrm{C}$; subunits $\mathrm{A}$ and $\mathrm{B}$ are identical to the $\mathrm{C}$ and $\mathrm{D}$ subunits). Cys residues coordinating to $\mathrm{Fe}$ ions were deprotonated. His-274, 451, 297D, 359D and 519D were assumed to be protonated on the ND1 atom, His-31, 196, 285, $383,90 \mathrm{D}, 185 \mathrm{D}, 363 \mathrm{D}$ and 457D were protonated on both the ND1 and NE2 atoms (and therefore positively charged), whereas the remaining $14 \mathrm{His}$ residues were modelled with a proton on the NE2 atom. The homocitrate was modelled in the singly protonated state with a proton shared between the hydroxyl group (which coordinates to Mo) and the $\mathrm{O} 1$ carboxylate atom. This protonation state was found to be the most stable in a recent extensive $\mathrm{QM} / \mathrm{MM}$, molecular dynamics and quantumrefinement study ${ }^{30}$ and it is also supported by another study. ${ }^{32}$

The protein was solvated in a sphere with a radius of $65 \AA$ around the geometrical centre of the protein. $160 \mathrm{Cl}^{-}$and 182 $\mathrm{Na}^{+}$ions were added to neutralise the protein and give an ionic strength of $0.2 \mathrm{M}^{33}$ The final system contained 133915 atoms. The added protons, counter ions and water molecules were optimised by a simulated annealing calculation (up to $370 \mathrm{~K}$ ), followed by a minimisation, keeping the other atoms fixed at the crystal-structure positions. ${ }^{30}$

All MM calculations were performed with the Amber software. ${ }^{34}$ For the protein, we used the Amber ff14SB force field ${ }^{35}$ and water molecules were described by the TIP3P model. ${ }^{36}$ For the metal sites, the MM parameters were the same as in our previous investigation. ${ }^{30}$ The metal sites were treated by a nonbonded model ${ }^{37}$ and charges were obtained with the restrained electrostatic potential method, obtained at the TPSS/def2-SV(P) level of theory $^{38,39}$ and sampled with the Merz-Kollman scheme. ${ }^{40}$

\section{QM/MM calculations}

The QM/MM calculations were performed with the ComQum software. ${ }^{41,42}$ In this approach, the protein and solvent are split into two subsystems: system 1 (the QM region) was relaxed by QM methods, whereas system 2 contained the remaining part of the protein and the solvent. It was kept fixed at the original coordinates (equilibrated crystal structure). ${ }^{30}$

In the QM calculations, system 1 was represented by a wavefunction, whereas all the other atoms were represented by an array of partial point charges, one for each atom, taken from the MM setup. When there is a bond between systems 1 and 2, the hydrogen link-atom approach was employed: the QM system was capped with hydrogen atoms (hydrogen link atoms, HL), the positions of which are linearly related to the corresponding carbon atoms (carbon link atoms, CL) in the full system. ${ }^{41,43}$ All MM atoms were included in the point-charge model, except the CL atoms. ${ }^{44}$

The total QM/MM energy in ComQum was calculated as ${ }^{41,42}$

$$
E_{\mathrm{QM} / \mathrm{MM}}=E_{\mathrm{QM} 1+\mathrm{ptch} 2}^{\mathrm{HL}}+E_{\mathrm{MM} 12, q_{1}=0}^{\mathrm{CL}}-E_{\mathrm{MM} 1, q_{1}=0}^{\mathrm{HL}}
$$

where $E_{\mathrm{QM} 1+p t c h}^{\mathrm{HL}}$ is the QM energy of the QM system truncated by $\mathrm{HL}$ atoms and embedded in the set of point charges modelling system 2 (but excluding the self-energy of the point charges). $E_{\mathrm{MM} 1, q_{1}=0}^{\mathrm{HL}}$ is the MM energy of the QM system, still truncated by $\mathrm{HL}$ atoms, but without any electrostatic interactions. Finally, $E_{\mathrm{MM} 12, q_{1}=0}^{\mathrm{CL}}$ is the classical energy of all atoms in the system with CL atoms and with the charges of the QM region set to zero (to avoid double-counting of the electrostatic interactions). Thus, ComQum employs a subtractive scheme with electrostatic embedding and van der Waals link-atom corrections. ${ }^{45}$ The geometry optimisations were continued until the energy change between two iterations was less than $2.6 \mathrm{~J} \mathrm{~mol}^{-1}\left(10^{-6}\right.$ a.u.) and the maximum norm of the Cartesian gradients was below $10^{-3}$ a.u.

\section{QM calculations}

All QM calculations were performed with the Turbomole software (versions 7.1 and 7.2). ${ }^{46}$ We employed 13 different DFT methods, TPSS $^{38}$ PBE $^{47}$ BP86, ${ }^{48,49}$ BLYP,${ }^{48,50}$ B97D,${ }^{51}$ PBE0, ${ }^{52}$ TPSS $^{53}$ B3LYP ${ }^{48,50,54}$ BHLYP, ${ }^{55}$ M06, ${ }^{56}$ M06-L, ${ }^{57}$ M06-2X, ${ }^{56}$ and M06$\mathrm{HF}^{58}$ TPSS, PBE, BP86, BLYP, B97D and M06-L are (meta-) generalized gradient approximation (GGA) functionals, with no admixture of Hartree-Fock (HF) exchange. The other DFT methods are hybrid functionals with 10\% (TPSSh), 20\% (B3LYP), 25\% (PBE0), 27\% (M06), 50\% (BHLYP), 54\% (M06-2X), and $100 \%$ (M06-HF) HF exchange (\% HF). All calculations employed the def2-SV(P) basis set, ${ }^{39}$ because previous studies have shown that increasing the basis set to def2-TZVPD changes the relative energies by less than $16 \mathrm{~kJ} \mathrm{~mol}^{-1} \cdot{ }^{29,31}$ The calculations were sped up by expanding the Coulomb interactions in an auxiliary basis set, the resolution-of-identity approximation. ${ }^{59,60}$ 
Empirical dispersion corrections were included with the DFT-D3 approach $^{61}$ and Becke-Johnson damping, ${ }^{62}$ using parameters optimised for each DFT method, as implemented in Turbomole for all functionals except M06, M06-L, M06-2X and M06-HF (for which no parameters are available because the methods should include dispersion effects by parameterisation).

It should be noted that the M06-2X and M06-HF functionals were constructed for main-group thermochemistry and chargetransfer excitations, respectively, and are not recommended for systems with significant static correlation, like transition metals. ${ }^{56}$ The same applies also to BHLYP with its large amount of HF exchange. ${ }^{63}$ These three methods reproduce the structure of the resting state quite poorly (Fig. S3, ESI $\dagger$ ) and they often

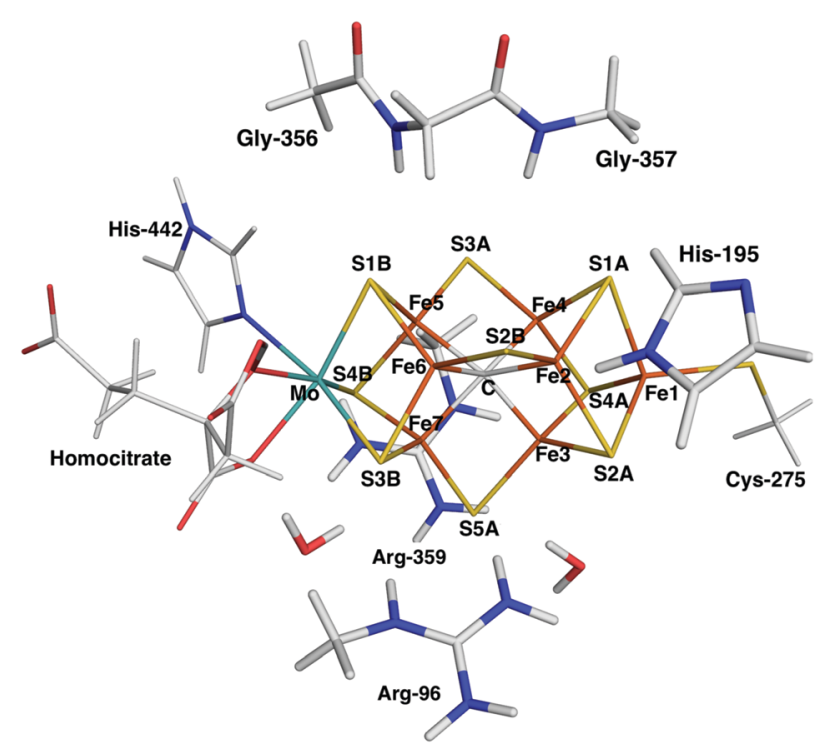

Fig. 1 The FeMo cluster illustrating the QM system used in all QM/MM calculations and the naming of the atoms in the cluster. give rise to a qualitative change in the geometry of the optimised structures of models 5-7 and 9 (Table S1, ESI $\dagger$ ). Therefore, these three functionals were excluded from the figures in the main article, but the results are included in the ESI $\dagger$ (Fig. S1-S3), because they emphasize the trends with respect to \% $\mathrm{HF}$.

The FeMo cluster was modelled by $\mathrm{MoFe}_{7} \mathrm{~S}_{9} \mathrm{C}$ (homocitrate) $\left(\mathrm{CH}_{3} \mathrm{~S}\right)$ (imidazole), where the last two groups are models of Cys-275 and His-442. In addition, all groups that form hydrogen bonds to the FeMo cluster within $3.5 \AA$ in the crystal structure ${ }^{7}$ were also included, viz. Arg-96, His-195 and Arg-359 (sidechains), Gly-356, Gly-357 (backbone), as well as two water molecules. In total, the QM system contained 113-117 atoms (depending on the $\mathrm{E}_{n}$ state, i.e. the number of added protons) and it is shown in Fig. 1. Following recent Mössbauer, anomalous dispersion and QM investigations, ${ }^{13,24,64,65}$ we used the oxidation stateassignment $\mathrm{Mo}^{\mathrm{III}} \mathrm{Fe}_{3}^{\mathrm{II}} \mathrm{Fe}_{4}^{\mathrm{III}}$ of the metal ions in the resting $\left(\mathrm{E}_{0}\right)$ state, giving a net charge of -5 for the QM system. We studied three oxidation states of the FeMo cluster, obtained by adding 0,2 or 4 electrons to the resting states, denoted $\mathrm{E}_{0}, \mathrm{E}_{2}$ and $\mathrm{E}_{4}$. One proton was added together with each electron, so the net charge of the cluster was -3 for all three states.

Experiments have shown that the ground spin state of $\mathrm{E}_{0}$ and $E_{2}$ are quartets with a surplus of three $\alpha$ electrons, whereas $E_{4}$ is a doublet. ${ }^{3,13}$ These spin states were used in all calculations. The electronic structure of all QM calculations was obtained with the broken-symmetry (BS) approach: ${ }^{18}$ for each model, we used the best of the 35 possible BS states ${ }^{31}$ found in our previous study. ${ }^{29}$ These states are specified in Table 1 , which also give further description of the various models (which atoms are protonated and the number of bonds within the cluster). The 13 calculations with different DFT methods for each complex were started with the same wavefunction, to ensure that they belong to the same BS state. Bond lengths involving the added protons are described in Table S2 (ESI $\dagger$ ) for all models optimised with the 13 DFT methods, spin densities on the metals are

Table 1 Description of the ten models, listing the $E_{n}$ state, the positions of the added protons (atom names are shown in Fig. 1; the number in brackets indicates the direction of the proton ${ }^{a 27}$ ), the BS state, ${ }^{b}$ the number of bonds of various types (a Fe-Fe interaction is assumed to be broken if it is $0.5 \AA$ longer than in the resting state; the $\mathrm{Fe}-\mathrm{S}$ and $\mathrm{Fe}-\mathrm{C}$ bonds are considered broken if they are $0.3 \AA$ longer than in the resting state), and the number of Fe( $I$ ) ions in the FeMo cluster ( $n$, formal assignment, assuming that all metal-bound hydrogen atoms are hydride ions)

\begin{tabular}{|c|c|c|c|c|c|c|c|c|c|c|c|c|c|c|}
\hline & \multirow[b]{2}{*}{$\mathrm{E}$} & \multicolumn{4}{|c|}{ Position of the $\mathrm{H}$ atoms ${ }^{a}$} & \multirow{2}{*}{$\begin{array}{l}\text { BS } \\
\text { State }^{b}\end{array}$} & \multicolumn{4}{|c|}{ \# $\mathrm{Fe}-\mathrm{X}$ bonds } & \multicolumn{3}{|c|}{ \# other bonds } & \multirow[b]{2}{*}{$n$} \\
\hline & & H1 & $\mathrm{H} 2$ & H3 & $\mathrm{H} 4$ & & $\mathrm{Fe}$ & $\mathrm{S}$ & $\mathrm{C}$ & $\mathrm{H}$ & $\mathrm{S}-\mathrm{H}$ & $\mathrm{C}-\mathrm{H}$ & $\mathrm{H}-\mathrm{H}$ & \\
\hline 0 & $\mathrm{E}_{0}$ & & & & & BS7-1 & 12 & 22 & 6 & 0 & 0 & 0 & 0 & 3 \\
\hline 1 & $\mathrm{E}_{4}$ & S2B(3) & $\mathrm{C}(2367)$ & $\mathrm{C}(3457)$ & $C(2456)$ & BS2 & 4 & 21 & 2 & 0 & 1 & 3 & 0 & 7 \\
\hline 2 & $\mathrm{E}_{4}$ & $\mathrm{~S} 2 \mathrm{~B}(3)$ & $\mathrm{C}(2367)$ & $\mathrm{C}(3457)$ & $\mathrm{S} 2 \mathrm{~A}(\mathrm{Fe} 1)$ & BS2 & 8 & 21 & 3 & 0 & 2 & 2 & 0 & 7 \\
\hline 3 & $\mathrm{E}_{4}$ & $\mathrm{~S} 2 \mathrm{~B}(3)$ & $\mathrm{C}(2367)$ & $\mathrm{Fe} 6 / 7$ & S2A(Mo) & BS10-1 & 9 & 21 & 5 & 1 & 2 & 1 & 0 & 5 \\
\hline 4 & $\mathrm{E}_{4}$ & $\mathrm{~S} 2 \mathrm{~B}(3)$ & $\mathrm{Fe} 2 / 6(5)$ & S5A(2) & $\mathrm{Fe} 3 / 7(2)$ & BS9-2 & 12 & 22 & 6 & 2 & 2 & 0 & 0 & 3 \\
\hline 5 & $\mathrm{E}_{4}$ & $\mathrm{~S} 2 \mathrm{~B}(3)$ & $\mathrm{Fe} 2 / 6(3)$ & $\mathrm{Fe} 4$ & Fe5 & BS6-2 & 12 & 22 & 6 & 3 & 1 & 0 & 0 & 1 \\
\hline 6 & $\mathrm{E}_{4}$ & $\mathrm{~S} 2 \mathrm{~B}(3)$ & $\mathrm{Fe} 2 / 6(3)$ & $\mathrm{Fe} 5^{c}$ & $\mathrm{H}_{2}$ & BS7-3 & 12 & 22 & 6 & 2 & 1 & 0 & 1 & 3 \\
\hline 7 & $\mathrm{E}_{4}$ & $\mathrm{~S} 2 \mathrm{~B}(3)$ & $\mathrm{Fe} 2^{d}$ & $\mathrm{Fe} 4$ & $\mathrm{Fe} 2^{d}$ & BS7-3 & 12 & 22 & 6 & 3 & 1 & 0 & 1 & 3 \\
\hline 8 & $\mathrm{E}_{2}$ & $\mathrm{C}(3457)$ & $\mathrm{C}(2367)$ & & & BS8-6 & 7 & 22 & 4 & 0 & 0 & 2 & 0 & 5 \\
\hline 9 & $\mathrm{E}_{2}$ & $\mathrm{~S} 2 \mathrm{~B}(3)$ & $\mathrm{Fe} 2 / 6(3)$ & & & BS7-3 & 12 & 22 & 6 & 1 & 1 & 0 & 0 & 3 \\
\hline
\end{tabular}

${ }^{a} \mathrm{~S} 2 \mathrm{~B}(3)$ means that the proton on S2B points towards S3A; C(2367) means that the proton is on the Fe2-Fe3-Fe6-Fe7 face and similar for C(3457) and C(2456); S2A(Fe1) and S2A(Mo) mean that the proton on S2A points towards Fe1 or Mo, respectively; Fe2/6(5) or (3) means that the hydride ion bridging Fe2 and Fe6 points towards S5A or S3A; S5A(2) means that the proton on S5A points towards S2B; Fe3/7(2) means that the hydride ion bridging Fe3 and Fe7 points towards S2B; hydride ions terminally bound to one Fe ion are trans to the central carbide ion. ${ }^{b}$ The BS states are defined by which three Fe ions have a negative spin, ${ }^{31}$ viz. Fe2, 3, and 5 for BS7-1, Fe2, 3 and 4 for BS2, Fe1, 2 and 5 for BS10-1, Fe1, 3 and 7 for BS9-2, Fe1, 5 and 7 for BS6-2, Fe3, 4 and 6 for BS7-3 and Fe3, 4 and 5 for BS8-6. ${ }^{c} \mathrm{H}_{2}$ bound end-on to Fe5. ${ }^{d} \mathrm{H}_{2}$ bound side-on to Fe2. 
collected in Table S6 (ESI $\dagger$ ), whereas Table S7 (ESI $\dagger$ ) list the coordinates for the complexes.

Undoubtedly, the present calculations involve several approximations, e.g. the use of single QM/MM structures and small basis sets without any inclusion of entropy or thermal effects. However, the extremely large difference between the DFT results will undoubtedly remain also in improved calculations.

\section{Results}

In this study, we compare energies and geometries of 10 models of the FeMo cluster in the $\mathrm{E}_{4}, \mathrm{E}_{2}$ or $\mathrm{E}_{0}$ state, shown in Fig. 2, calculated with 13 different DFT methods. All structures were optimised with QM/MM individually for each DFT method. The aim is to describe and understand the large variation of the results obtained with the various methods and to gain some understanding of which method gives the more reliable results.

\section{$\mathbf{E}_{4}$ states}

We first compare the relative stability of the best protonation states of $\mathrm{E}_{4}$ obtained with either the B3LYP (1) or TPSS (5) functionals in our extensive study, $\Delta E_{15}{ }^{29}$ As can be seen from Fig. 2, both structures are protonated on one of the $\mu_{2}$ sulfide ions (S2B), whereas the other three protons are on the central carbide ion in 1 but on Fe ions in 5. Protonation of the carbide leads to significant distortions of the FeMo cluster ( $c f$. Table 1), but the carbide ion remains inside the cluster in our $\mathrm{QM} / \mathrm{MM}$ calculations, ${ }^{29}$ in variance to the QM-cluster calculations by Siegbahn, in which the protonated carbide ion moves to the periphery of the cluster. ${ }^{66}$

From the relative energies in Fig. 3 (blue curve), it can be seen that the (meta) GGA functionals (TPSS, PBE, BP86, BLYP, M06-L and B97D) predict that structure 5 is $8-242 \mathrm{~kJ} \mathrm{~mol}^{-1}$ more stable than structure 1. On the other hand, the hybrid functionals (TPSSh, B3LYP, PBE0 and M06) predict that structure $\mathbf{1}$ is $39-353 \mathrm{~kJ} \mathrm{~mol}^{-1}$ more stable than structure $\mathbf{5}$. The latter agrees with Siegbahn's suggestion (based on B3LYP calculations) that it is much more favourable to protonate the central carbide than the metal ions. ${ }^{28}$ However, a similar study based on a GGA functional would have reached the opposite conclusion.

Thus, the various DFT methods give $\Delta E_{15}$ results that differ by up to $595 \mathrm{~kJ} \mathrm{~mol}^{-1}$. This is $4-30$ times larger variation than

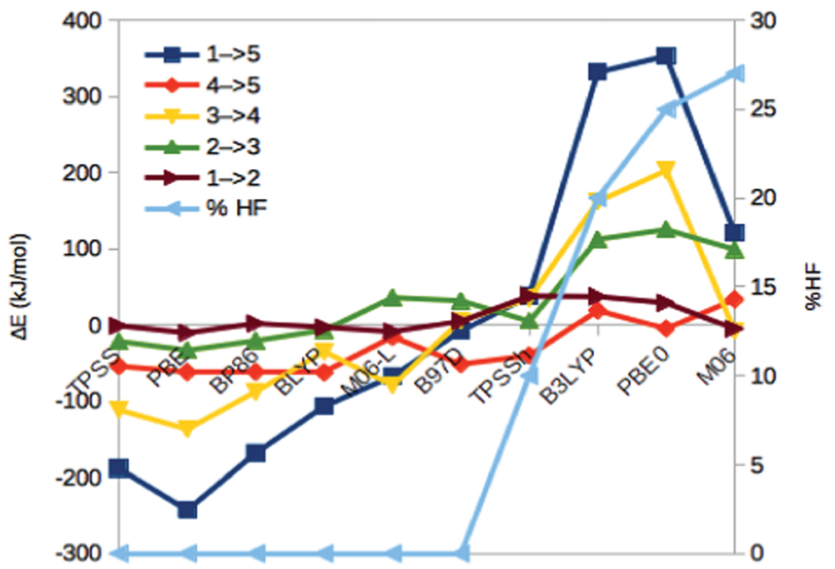

Fig. 3 Energies for the $1 \rightarrow 5,4 \rightarrow 5,3 \rightarrow 4,2 \rightarrow 3$ and $1 \rightarrow 2$ isomerisations, obtained with ten different DFT methods. The cyan line shows \% HF for the various methods (right axis).

what is found in other systems. ${ }^{67-69}$ For example, Fig. 4 shows the reaction energies of the $\mathrm{N}_{2}+3 \mathrm{H}_{2} \rightarrow 2 \mathrm{NH}_{3}$ reaction, divided into three steps (involving the formation of $\mathrm{N}_{2} \mathrm{H}_{2}, \mathrm{~N}_{2} \mathrm{H}_{4}$ and finally two $\mathrm{NH}_{3}$ molecules). It can be seen that the 13 DFT functionals give results that agree within $35-38 \mathrm{~kJ} \mathrm{~mol}^{-1}$. In particular, all methods agree that the first step (formation of $\mathrm{N}_{2} \mathrm{H}_{2}$ ) is strongly uphill (by $167 \mathrm{~kJ} \mathrm{~mol}^{-1}$ on average), whereas the other two steps are downhill by 102 and $154 \mathrm{~kJ} \mathrm{~mol}^{-1}$ on average, respectively.

The hybrid functionals involve a varying amount of HartreeFock exchange (\% HF, 10-27\%), as is also shown in Fig. 3 (cyan line and right axis). There is a fair correlation between $\Delta E_{15}$ and $\% \mathrm{HF}, R^{2}=0.75$, although M06 breaks the trend. In fact, we also used three functionals with a larger \% $\mathrm{HF}$, which increased the correlation to 0.88 and the energy range to $1097 \mathrm{~kJ} \mathrm{~mol}^{-1}$ (Fig. S1, ESI $\dagger$ ), but since such methods are normally not recommended for transition-metal systems ${ }^{56,63}$ and they gave qualitative changes in the structure of $\mathbf{5}$, we present these results in the ESI. $\dagger$ For the $\mathrm{N}_{2}+3 \mathrm{H}$ reactions, the correlation to the \% HF is appreciably weaker, $0.03-0.42$.

If the structures are not optimized with each DFT functional, the variation is even larger, $862 \mathrm{~kJ} \mathrm{~mol}^{-1}$ (using the B3LYP structure for 1 and the TPSS structure for 5; Table S3, ESI $\dagger$ ), showing the importance of optimizing the individual structures

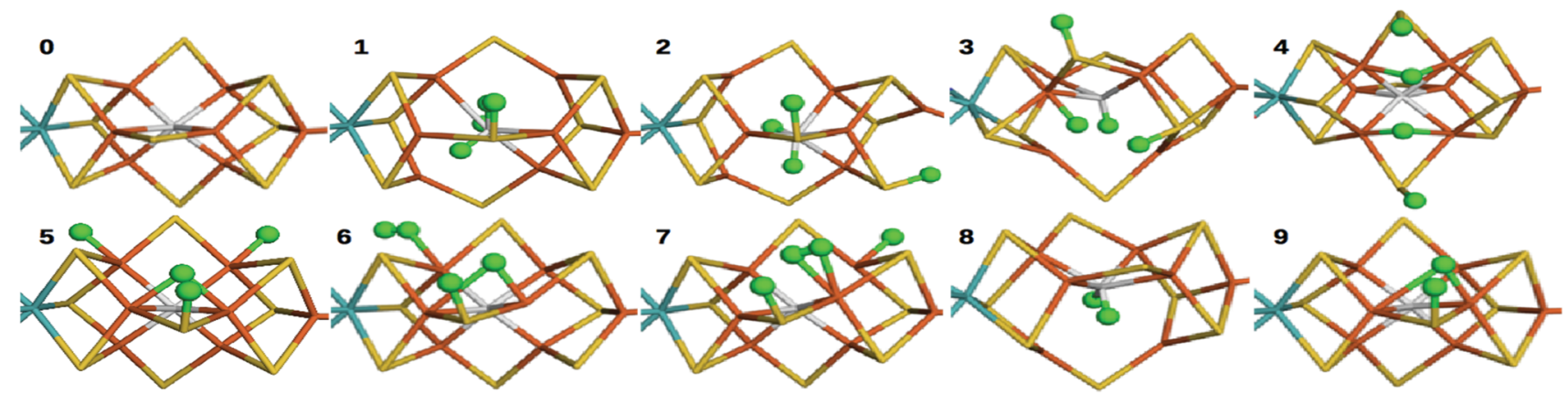

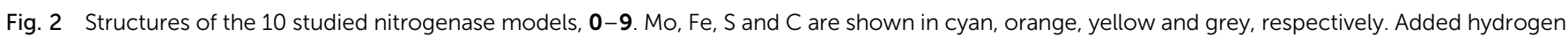
atoms are shown in green balls. For clarity, only the central atoms in the cluster are shown. 


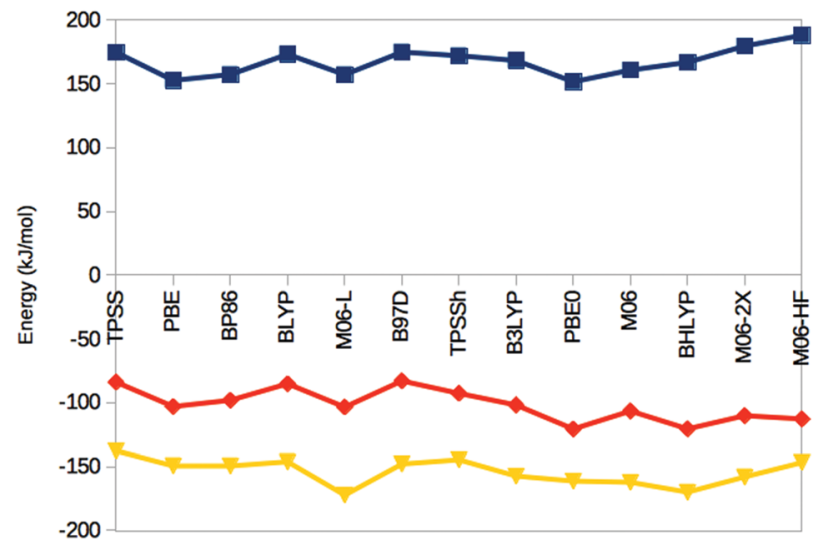

Fig. 4 Reaction energies for the $\mathrm{N}_{2}+\mathrm{H}_{2} \rightarrow \mathrm{N}_{2} \mathrm{H}_{2}$ (top, blue), $\mathrm{N}_{2} \mathrm{H}_{2}+$ $\mathrm{H}_{2} \rightarrow \mathrm{N}_{2} \mathrm{H}_{4}$ (middle, red) and $\mathrm{N}_{2} \mathrm{H}_{4}+\mathrm{H}_{2} \rightarrow 2 \mathrm{NH}_{3}$ (bottom, yellow) reactions, obtained in the gas phase with the 13 DFT methods.

when the energy differences are so large. However, the correlation of $\Delta E_{15}$ obtained with or without geometry optimization is very high, 0.98 .

In 1, there are three protons on the central carbide, whereas in $\mathbf{5}$, they are instead (as hydride ions) on Fe ions. To further understand the large variation in the DFT results, we did similar calculations on three structures $(2,3$ and 4$)$ with intermediate protonation, viz. with 2, 1 and no protons on the carbide and with 0,1 and 2 metal-bound hydride ions, respectively ( $c f$. Fig. 2). In fact, structure 4 is one of the $E_{4}$ structures suggested by the Hoffman group (based on BP86 calculations) with two hydride ions bridging two Fe ions each and two protons on sulfide ions. ${ }^{27}$ From Fig. 3 (red line), it can be seen that $\Delta E_{45}$ is similar for most functionals, with 5 being more stable than 4 by 5-62 $\mathrm{kJ} \mathrm{mol}^{-1}$. Only B3LYP and M06 give the opposite ordering (by $19-34 \mathrm{~kJ} \mathrm{~mol}^{-1}$ ). However, there is a quite strong correlation between $\Delta E_{45}$ and \% HF, $R^{2}=0.8$.

In model 3, there is only one hydride ion and the carbide ion is singly protonated. Interestingly, $\Delta E_{34}$ shows an appreciably larger variation among the functionals (Fig. 3, yellow). In general, $\mathbf{3}$ is less stable than $\mathbf{4}$ according to the GGA functionals (by $35-137 \mathrm{~kJ} \mathrm{~mol}^{-1}$ ), whereas the opposite is true with the hybrid functionals (by 35-203 $\mathrm{kJ} \mathrm{mol}^{-1}$ ). However, with B97D and M06, they are of a similar energy. There is a fair correlation between $\Delta E_{34}$ and $\% \operatorname{HF}\left(R^{2}=0.58 ; 0.76\right.$ with all 13 functionals).

Finally, model 2 has no hydride ions and a doubly protonated carbide ion. From Fig. 3 (green line), it can be seen that the GGA functionals, except M06-L and B97D indicate that 3 is $7-33 \mathrm{~kJ} \mathrm{~mol}^{-1}$ more stable than 2 , whereas the other methods give the opposite result by $5-125 \mathrm{~kJ} \mathrm{~mol}^{-1}$. There is a strong correlation between $\Delta E_{23}$ and $\% \mathrm{HF}\left(R^{2}=0.78\right.$ or 0.92$)$. If we instead compare the stability of structures $\mathbf{2}$ and 1, i.e. the effect of moving a proton from a sulfide to the carbide (brown line in Fig. 3), we find that the GGA functionals and M06 indicate that structures $\mathbf{2}$ and $\mathbf{1}$ are close in energy $\left(-10\right.$ to $\left.2 \mathrm{~kJ} \mathrm{~mol}^{-1}\right)$, whereas the other hybrid functionals indicate that structure $\mathbf{1}$ is $29-39 \mathrm{~kJ} \mathrm{~mol}^{-1}$ more stable than structure 2 . There is only a low correlation between $\Delta E_{12}$ and $\% \operatorname{HF}(0.3$, but it increases to 0.75 with all 13 DFT functionals).
Thus, the large variation in $\Delta E_{15}$ among the DFT functionals is caused mainly by $\Delta E_{34}$ and $\Delta E_{23}$. This indicates that the variation is related to at least two factors. The isomerizations $4 \rightarrow 5,3 \rightarrow 4$ and $2 \rightarrow 3$ all involve a change in the number of hydride ions and therefore also the formal oxidation state of the Fe ions (a hydride ion has two more electrons than a proton; therefore, the number of Fe(II) ions in the structures 1-5 are 7, 7, 5, 3 and 1, respectively), so that structures $\mathbf{1}$ and $\mathbf{5}$ actually differ by six levels in the formal oxidation state of the metal ions. On the other hand, $\mathbf{3} \rightarrow \mathbf{4 , 2} \rightarrow \mathbf{3}$ and $\mathbf{1} \rightarrow \mathbf{2}$ involve the formation of $\mathrm{C}-\mathrm{H}$ bonds, associated with a distortion of the FeMo cluster, leading to partial cleavage of $\mathrm{Fe}-\mathrm{C}, \mathrm{Fe}-\mathrm{S}$ and $\mathrm{Fe}-\mathrm{Fe}$ bonds. This is detailed in Table 1, in which it can be seen that eight of the Fe-Fe distances have elongated by more than $0.5 \AA$ and four of the Fe-C bonds and one of the Fe-S bonds are elongated by more than $0.3 \AA$ in $\mathbf{1}$, compared to 5 or the resting $E_{0}$ state. Models 2 and 3 have a lower number of broken bonds ( $c$. Table 1). Apparently, the variation among the DFT methods is the largest when both effects apply.

\section{$\mathrm{H}_{2}$ binding and dissociation}

To gain some additional information about how the energies vary with the DFT functionals, we studied also the binding and dissociation of $\mathrm{H}_{2}$ from the $\mathrm{E}_{4}$ and $\mathrm{E}_{2}$ states of the MoFe cluster. First, we included two complexes with $\mathrm{H}_{2}$ bound to the FeMo cluster in the $\mathrm{E}_{4}$ state, both with a proton on S2B. Structure 6 has $\mathrm{H}_{2}$ bound end-on to Fe5 and the fourth $\mathrm{H}$ is bridging $\mathrm{Fe} 2$ and $\mathrm{Fe} 6$, whereas in structure $7, \mathrm{H}_{2}$ is bound side-on to $\mathrm{Fe} 2$ and with the fourth $\mathrm{H}$ on $\mathrm{Fe} 4$ ( $c f$. Fig. 2; atom names are shown in Fig. 1). For all methods, end-on binding (6) was more favourable than side-on binding (7), by $20-88 \mathrm{~kJ} \mathrm{~mol}^{-1}$ (Fig. 5, blue line). Moreover, with the GGA functionals, 6 was more stable than 5 (the most stable $\mathrm{E}_{4}$ structure with those functionals) by 7-104 kJ mol ${ }^{-1}$ (Fig. 5, red line). On the other hand, 1 was appreciably more stable than $\mathbf{6}$ for the B3LYP and PBE0 functionals (by 239-246 kJ mol${ }^{-1}$ ), whereas the two structures were isoenergetic within $2 \mathrm{~kJ} \mathrm{~mol}^{-1}$ with TPSSh and M06.

Next, we studied two structures of the $\mathrm{E}_{2}$ state (i.e. with two protons and electrons less), one with the two protons on the

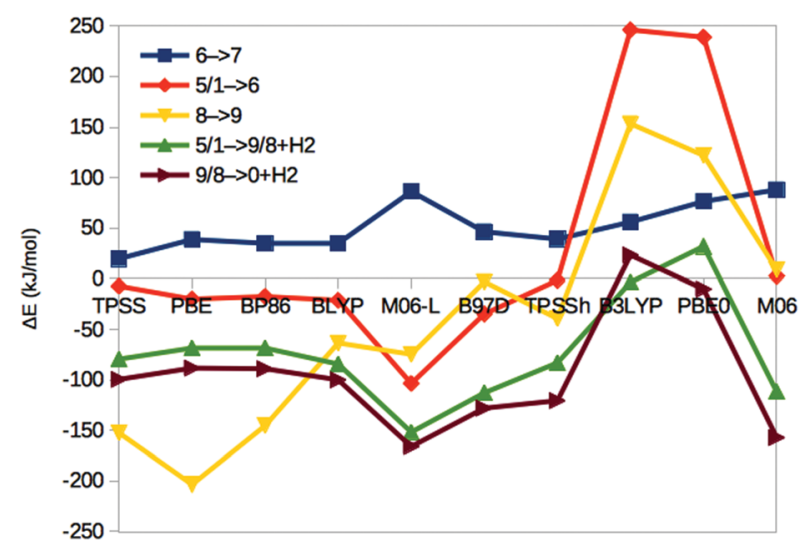

Fig. 5 Energies for the $\mathbf{6} \rightarrow \mathbf{7 , 5 / 1} \rightarrow \mathbf{6 , 8} \rightarrow \mathbf{9 , 5 / 1} \rightarrow \mathbf{9 / 8}+\mathrm{H}_{2}$ and $\mathbf{9 / 8} \rightarrow$ $0+\mathrm{H}_{2}$ reactions, obtained with ten different DFT methods (using the structure with the lowest energy for $5 / \mathbf{1}$ and $\mathbf{9 / 8}$ for each method). 
central carbide (8) and the other with one proton on the $\mathrm{S} 2 \mathrm{~B} \mu_{2}$ sulfide and one hydride ion bridging $\mathrm{Fe} 2$ and Fe6 (9; $c f$. Fig. 2). They are the best $\mathrm{E}_{2}$ structures obtained in our previous study with B3LYP and TPSS, respectively. ${ }^{29}$ As expected, the GGA functionals, but also TPSSh, suggest that 9 is $3-203 \mathrm{~kJ} \mathrm{~mol}^{-1}$ more stable than $\mathbf{8}$, whereas the other hybrid functionals favour 8 by 9 (M06) or $122-153 \mathrm{~kJ} \mathrm{~mol}^{-1}$ (Fig. 5, yellow line). The correlation of $\Delta E_{89}$ to $\% \mathrm{HF}$ is 0.6 .

With these structures, we can estimate the dissociation energy of $\mathrm{H}_{2}$ from either 5 or 1. It is favourable for all GGA functionals $\left(5 \rightarrow 9+\mathrm{H}_{2}\right.$ ) by $68-152 \mathrm{~kJ} \mathrm{~mol}^{-1}$. For the hybrid functionals ( $\mathbf{1} \rightarrow \mathbf{8}+\mathrm{H}_{2}$ ), $\mathrm{H}_{2}$ dissociation is also favourable by 3-112 kJ mol ${ }^{-1}$, except for PBE0 (Fig. 5, green line).

Finally, we studied also the resting state of the enzyme $\left(\mathrm{E}_{0} ; 0\right)$ to obtain an estimate of the dissociation energy of $\mathrm{H}_{2}$ from $\mathrm{E}_{2}\left(\mathbf{9} / \mathbf{8} \rightarrow \mathbf{0}+\mathrm{H}_{2}\right)$. Fig. 5 (brown line) shows that it is favourable for all GGA functionals, as well as TPSSh and M06 by 88-166 kJ mol ${ }^{-1}$, whereas it is unfavourable for B3LYP and nearly thermoneutral for PBE0.

\section{Geometry of the resting state}

For $\mathrm{E}_{0}$, we can also compare the $\mathrm{QM} / \mathrm{MM}$ structures with the crystal structure of nitrogenase $(3 \mathrm{U} 7 \mathrm{Q}) .{ }^{7}$ It is at such a high resolution $(1.0 \AA)$ that it can be used to calibrate the DFT geometries. In fact, it has been used to determine the brokensymmetry state of the FeMo cluster and the protonation of the homocitrate ligand. ${ }^{32}$ Fig. 6 shows the root-mean-squared deviation (RMSD), as well as the mean absolute deviation (MAD) and maximum deviation of the metal-ligand and metal-metal distances for the various DFT methods. It can be seen that TPSSh gives the lowest RMSD (0.06 $\AA$ ), whereas the best metal-ligand distances are obtained with PBE and BP86 (giving the lowest MAD $=0.02 \AA$ ) or BLYP (lowest maximum deviation, $0.06 \AA$ ). On the other hand, the best metal-metal distances are obtained with B97D and TPSSh (MAD = $0.02 \AA)$ or BLYP methods (maximum deviation of $0.07 \AA$ ). Thus, TPSSh

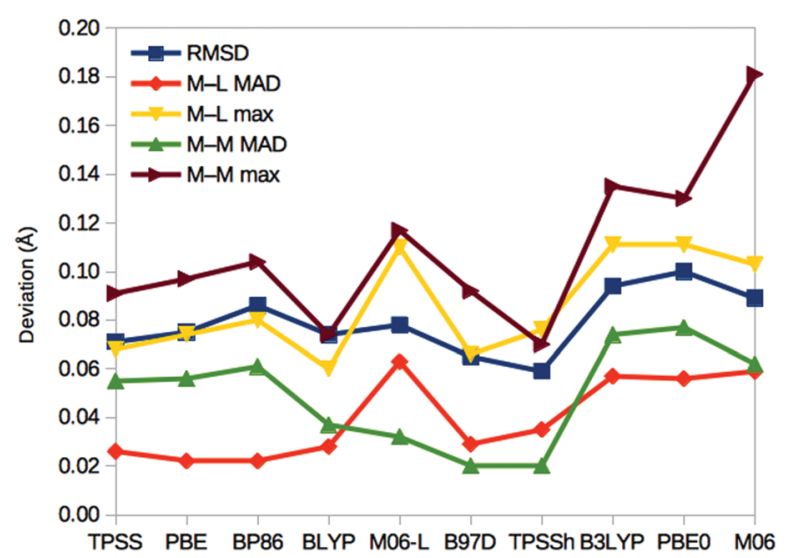

Fig. 6 Performance of the various DFT methods for the structure of the resting $E_{0}$ state compared to the crystal structure. ${ }^{6}$ For each method, the RMSD for the Mo, Fe, S and $\mathrm{C}$ atoms of the cluster, as well as the directly connected ligand $\mathrm{S}, \mathrm{O}$ and $\mathrm{N}$ atoms are given, together with the MAD and maximum deviation for the 34 metal-ligand $(M-L)$ distances and the 15 short $(<3 \AA$ ) metal-metal $(M-M)$ distances. and the GGA functionals (except M06-L) give the best structures, appreciably better than those of the other hybrid functionals.

\section{Spin densities}

The geometry optimisations with different DFT functionals were started from the same wavefunction, to keep them as similar as possible. We used the experimentally observed spin state and the best BS state obtained for each structure in our previous investigation (listed in Table 1). ${ }^{29}$ These were obtained by B3LYP for structures 1-3 and 8, and by TPSS for the other structures. Thus, we did not attempt to find better BS states for the other DFT functionals, which would be extremely timeconsuming and would have mixed in differences caused by the use of different BS states.

The spin densities obtained for the eight metal ions in the various calculations are listed in Table S6 (ESI $\dagger$ ). It can be seen that for most structures, the calculations with different DFT functionals remained in the same BS states, defined by the Fe ions with negative spin densities. However, for two structures, some DFT calculations gave qualitatively differences. For 6, most calculations were in the BS7-3 state, characterised with large negative spin populations on $\mathrm{Fe} 3, \mathrm{Fe} 4$ and $\mathrm{Fe} 6(\leq-2.4)$. However, the three GGA functionals PBE, BP86 and BLYP gave negative spin populations on the same three Fe ions, but that on Fe6 was small, -0.5 and Fe2 also had a negative spin of intermediate size, around -1.5 (therefore corresponding to the BS6-2 state). We could find the other state also with these functionals, but during geometry optimisation, they changed back to the original state, except for BLYP, for which it is $12 \mathrm{~kJ} \mathrm{~mol}^{-1}$ less stable than the original state. Still, this difference in the spin states is not reflected in the energies in Fig. 5.

For 4, all DFT calculations give large negative spin populations on Fe1 and Fe3. However, for five of the functionals (the GGA functionals, except M06-L), Fe7 had a small population on Fe7 $(-1.2$ for B97D or -0.1 for the others), whereas for the other functionals, the population is large and negative. Again, the other state could be found but it typically changed back during geometry optimisation. For BLYP, TPSSh and B3LYP, both states could be found, with energy differences of $12-97 \mathrm{~kJ} \mathrm{~mol}^{-1}$. In this case, the difference in the wavefunction is reflected in the energies in Fig. 3, which shows an increased stability for $\mathbf{4}$ for the functionals giving a high spin population on Fe7.

In addition, there are also systematic differences between the spin densities obtained with the various methods. First, the magnitude of the spin populations increases with \% HF. It is in general the lowest for BLYP, PBE and BP86 (2.5 on average) and the largest for PBE0 (3.6). The spin on the seven Fe ions also becomes more similar when \% HF increases. For example, the difference between the maximum and minimum absolute spin populations of structure $\mathbf{1}$ is $\mathbf{1 . 0}$ for PBE and BP86, but only 0.2 for PBE0.

The difference is even larger for the spin on the Mo ion. It is negative in all structures, except 5. With the TPSS, PBE, BP86 and BLYP functionals, it is in general small in magnitude, 0.2-0.5, but increased to 0.8-1.0 for structures 1 and 2. However, for M06-L and the hybrid functionals, it is appreciably higher, by $0.6-0.8$ 
on average. For structures $\mathbf{1}$ and 2, these functionals indicate a high-spin state of Mo (spin populations of 1.6-2.5), which are not consistent with ${ }^{95}$ Mo ENDOR experiments. ${ }^{70-72}$

\section{Discussion}

We have studied how the relative energies of various protonation and $\mathrm{H}_{2}$-bound states vary when they are calculated with ten different DFT methods. The results in Fig. 3 and 5 show an extremely large variation of the relative energies, up to $600 \mathrm{~kJ} \mathrm{~mol}^{-1}$, which often correlate to \% $\mathrm{HF}$ of the methods. A strong dependence on DFT for relative energies and a correlation to \% HF have been observed previously for several types of systems. For example, Reiher et al. studied the energy difference between the high- and low-spin states for a number of $\mathrm{Fe}(\mathrm{II}) \mathrm{S}_{4} \mathrm{NL}$ complexes with $\mathrm{L}=\mathrm{NH}_{3}, \mathrm{~N}_{2} \mathrm{H}_{4}, \mathrm{CO}$, $\mathrm{NO}^{+}, \mathrm{PH}_{3}$ and $\mathrm{PMe}_{3} .{ }^{67}$ They showed that predictions obtained by B3LYP and BP86 differed by $94-122 \mathrm{~kJ} \mathrm{~mol}^{-1}$ and that variants of B3LYP with \% HF varying between 0 and $25 \%$ gave energies that varied by up to $160 \mathrm{~kJ} \mathrm{~mol}^{-1}$. They observed that for $\% \mathrm{HF}$ between 10 and $15 \%$, the results agreed with experiments and recommended the B3LYP* method with $15 \%$ HF. This is the method used by Siegbahn for energies (but not structures) for nitrogenase. ${ }^{28}$

Likewise, for the homolytic $\mathrm{Co}(\mathrm{III})-\mathrm{C}$ bond dissociation energy in methyl corrin (coenzyme $\mathrm{B}_{12}$ without sidechains), Jensen and Ryde reported a difference of $57 \mathrm{~kJ} \mathrm{~mol}^{-1}$ between B3LYP and BP86 (51-66 kJ mol ${ }^{-1}$ for related models with different axial ligands and metals, Co, Fe or Ni). ${ }^{68}$ Later, more functionals were studied, increasing the variation to $139 \mathrm{~kJ} \mathrm{~mol}^{-1}$ (66 $\mathrm{kJ} \mathrm{mol}^{-1}$ excluding BHLYP) and showing a correlation to \% $\mathrm{HF}$ of $R^{2}=0.89 .^{73}$

For the oxy-transfer reaction in Mo-dependent dimethylsulfoxide reductase, Li et al. reported a variation of $116 \mathrm{~kJ} \mathrm{~mol}^{-1}$ for the activation energy and $103 \mathrm{~kJ} \mathrm{~mol}^{-1}$ for the reaction energy over eight DFT functionals (63 and $61 \mathrm{~kJ} \mathrm{~mol}^{-1}$ excluding BHLYP). The correlation to \% HF was 0.95 and $0.98 .^{74}$ The results were calibrated by using LCCSD(T0) calculations, which showed that for the activation energy, B3LYP gave the best result ( $9 \mathrm{~kJ} \mathrm{~mol}^{-1}$ error), whereas for the reaction energy, GGA functionals gave better results (errors of 1-6 $\mathrm{kJ} \mathrm{mol}^{-1}$ ). Similar results were obtained if geometries were optimised with the various methods or if also $\mathrm{W}$ was considered. ${ }^{69}$

Thus, previous studies of metalloenzymes have sometimes showed a large variation in the results of different DFT methods, especially for spin-state energies or when the oxidation state of the metal changes. However, the variation observed for nitrogenase is 4-10 times larger than what has been observed before.

Of course, the prime question is which of the DFT methods (if any) can be trusted. In general, hybrid DFT functionals give appreciably better results than GGA functionals for maingroup thermochemistry, e.g. with a weighted MAD of 21, 27 and $38 \mathrm{~kJ} \mathrm{~mol}^{-1}$ for M06-2X, B3LYP and TPSS, respectively, for the recent GMTKN55 test set (and even better results for double hybrid functionals). ${ }^{75}$ Hybrid functionals remain better for some transition-metal complexes, in particular with hard ligands. ${ }^{67,76}$ However, there are ample examples of transition-metal complexes (especially with softer ligands), for which GGA methods give better results than hybrid functionals, e.g. for reactions related to the nitrogenase reaction, ${ }^{77}$ for [NiFe]-hydrogenase models ${ }^{78,79}$ and for complexes with nitride and carbonyl ligands. ${ }^{80}$ In a recent test of 41 closed-shell organometallic reactions, PBE0 gave the best results among the DFT functionals employed in the present study, but the MAD was not much higher for TPSSh and TPSS (11, compared to 12 and $14 \mathrm{~kJ} \mathrm{~mol}^{-1}$ ), whereas B3LYP was appreciably worse, $21 \mathrm{~kJ} \mathrm{~mol}^{-1} \cdot{ }^{81}$ Clearly, the performance of DFT methods depend on the system studied, probably depending on the importance of static correlation. ${ }^{80}$

An alternative is to select the functional based on comparisons with experimental observations. In this study, we have compared several properties. Spectroscopic evidence indicate that the $\mathrm{E}_{4}$ state has two bridging hydride ions. ${ }^{3}$ Unfortunately, the results in Fig. 3 show that none of the DFT functionals suggest that the best $\mathrm{E}_{4}$ structure is 4 , which has two bridging hydride ions.

Moreover, experiments indicate that four electrons and protons should be added to the resting state before it may bind $\mathrm{N}_{2}$. Of course, it is then essential that $\mathrm{H}_{2}$ does not form and dissociate before $\mathrm{N}_{2}$ binds; otherwise two electrons and protons have been consumed with no gain. However, the energies in Fig. 5 indicate that all methods except B3LYP and PBE0 give strongly favourable $\mathrm{H}_{2}$ dissociation energies from both $\mathrm{E}_{4}$ and $\mathrm{E}_{2}$.

On the other hand, the results in Fig. 6 show that the TPSSh and GGA methods (except M06-L) best reproduce the crystal structure of the resting state. Thus, our calculations indicate that it may be hard to find a DFT method that gives good results for all properties of the FeMo cluster in nitrogenase.

\section{Conclusions}

We have shown that nitrogenase models with different protonation states give an unprecedentedly large variation in relative energies obtained with different DFT methods, up to $600 \mathrm{~kJ} \mathrm{~mol}^{-1}$. This variation is connected to differences in the formal oxidation state of the Fe ions in the cluster (number of hydride ions), as well as to the number of $\mathrm{Fe}-\mathrm{C}$ and $\mathrm{Fe}-\mathrm{H}$ bonds. This explains the large discrepancy between mechanisms suggested by different groups. ${ }^{27,28}$ Unfortunately, there is currently no quantum mechanical method that can be used to calibrate the DFT methods for a cluster of the size and electronic complexity as the one in nitrogenase. Instead, we suggest that energies are calculated with several DFT methods to check the sensitivity of the results. We have attempted to calibrate the methods by using experimental data. Unfortunately, GGA functionals and TPSSh give the best geometries, whereas B3LYP and PBE0 give better $\mathrm{H}_{2}$-dissociation energies but a high-spin Mo state for the best $\mathrm{E}_{4}$ structures. None of the tested methods indicate that the best $\mathrm{E}_{4}$ state has two bridging hydride groups. This calls for some humility in the interpretation of DFT results on nitrogenase. Undoubtedly, there is an urgent need of more accurate methods that can treat systems of the complexity of the FeMo cluster.

\section{Conflicts of interest}

There are no conflicts to declare. 


\section{Acknowledgements}

This work has been supported by grants from the Swedish Research Council (project 2014-5540), from COST through Action CM1305 (ECOSTBio), by the Royal Physiographic Society in Lund and by a scholarship to LC from the China Scholarship Council. The computations were performed on computer resources provided by the Swedish National Infrastructure for Computing (SNIC) at Lunarc at Lund University and HPC2N at Umeå University.

\section{References}

1 B. K. Burgess and D. J. Lowe, Chem. Rev., 1996, 96, 2983-3012.

2 B. Schmid, H.-J. Chiu, V. Ramakrishnan, J. B. Howard and D. C. Rees, in Handbook of Metalloproteins, John Wiley \& Sons, Ltd, 2006, pp. 1025-1036.

3 B. M. Hoffman, D. Lukoyanov, Z.-Y. Yang, D. R. Dean and L. C. Seefeldt, Chem. Rev., 2014, 114, 4041-4062.

4 B. E. Smith, Science, 2002, 297, 1654-1655.

5 J. Kim and D. C. Rees, Science, 1992, 257, 1677-1682.

6 O. Einsle, F. A. Tezcan, S. L. A. Andrade, B. Schmid, M. Yoshida, J. B. Howard and D. C. Rees, Science, 2002, 297, 1696.

7 T. Spatzal, M. Aksoyoglu, L. Zhang, S. L. A. Andrade, E. Schleicher, S. Weber, D. C. Rees and O. Einsle, Science, 2011, 334, 940.

8 T. Spatzal, K. A. Perez, O. Einsle, J. B. Howard and D. C. Rees, Science, 2014, 345, 1620-1623.

9 O. Einsle, J. Biol. Inorg. Chem., 2014, 19, 737-745.

10 R. R. Eady, Chem. Rev., 1996, 96, 3013-3030.

11 R. N. F. Thorneley and D. J. Lowe, in Molybdenum Enzymes, ed. T. G. Spiro, Wiley, New York, 1985, pp. 221-284.

12 K. M. Lancaster, M. Roemelt, P. Ettenhuber, Y. Hu, M. W. Ribbe, F. Neese, U. Bergmann and S. DeBeer, Science, 2011, 334, 974-977.

13 R. Bjornsson, F. A. Lima, T. Spatzal, T. Weyhermüller, P. Glatzel, E. Bill, O. Einsle, F. Neese and S. DeBeer, Chem. Sci., 2014, 5, 3096-3103.

14 F. Tuczek, in RSC Metallobiology Series 7, ed. R. Hille, C. Schulzke and M. L. Kirk, Royal Society of Chemistry, Cambridge, 2017, pp. 223-274.

15 I. Dance, J. Biol. Inorg. Chem., 1996, 1, 581-586.

16 K. K. Stavrev and M. C. Zerner, Int. J. Quantum Chem., 1998, 70, 1159-1168.

17 P. E. M. Siegbahn, J. Westerberg, M. Svensson and R. H. Crabtree, J. Phys. Chem. B, 1998, 102, 1615-1623.

18 T. Lovell, J. Li, T. Liu, D. A. Case and L. Noodleman, J. Am. Chem. Soc., 2001, 123, 12392-12410.

19 H. Xie, R. Wu, Z. Zhou and Z. Cao, J. Phys. Chem. B, 2008, 112, 11435-11439.

20 J. Kästner and P. E. Blöchl, J. Am. Chem. Soc., 2007, 129, 2998-3006.

21 P. P. Hallmen and J. Kästner, Z. Anorg. Allg. Chem., 2015, 641, 118-122.

22 I. Dance, Z. Anorg. Allg. Chem., 2015, 641, 91-99.

23 J. B. Varley, Y. Wang, K. Chan, F. Studt and J. K. Nørskov, Phys. Chem. Chem. Phys., 2015, 17, 29541-29547.
24 P. E. M. Siegbahn, J. Am. Chem. Soc., 2016, 138, 10485-10495. 25 M. L. McKee, J. Phys. Chem. A, 2016, 120, 754-764.

26 L. Rao, X. Xu and C. Adamo, ACS Catal., 2016, 6, 1567-1577. 27 D. Lukoyanov, N. Khadka, D. R. Dean, S. Raugei, L. C. Seefeldt and B. M. Hoffman, Inorg. Chem., 2017, 56, 2233-2240. 28 P. E. M. Siegbahn, J. Comput. Chem., 2018, 39, 743-747.

29 L. Cao and U. Ryde, J. Chem. Theory Comput., 2018, 14, 6653-6678.

30 L. Cao, O. Caldararu and U. Ryde, J. Phys. Chem. B, 2017, 121, 8242-8262.

31 L. Cao and U. Ryde, Int. J. Quantum Chem., 2018, 118, e25627.

32 B. Benediktsson and R. Bjornsson, Inorg. Chem., 2017, 56, 13417-13429.

33 B. M. Barney, J. Mcclead, D. Lukoyanov, M. Laryukhin, T. Yang, D. R. Dean, B. M. Hoffman and L. C. Seefeldt, Biochemistry, 2007, 46, 6784-6794.

34 D. A. Case, J. T. Berryman, R. M. Betz, D. S. Cerutti, T. E. Cheatham, T. A. Darden, R. E. Duke, T. J. Giese, H. Gohlke, A. W. Goetz, N. Homeyer, S. Izadi, P. Janowski, J. Kaus, A. Kovalenko, T. S. Lee, S. LeGrand, P. Li, T. Luchko, R. Luo, B. Madej, K. M. Merz, G. Monard, P. Needham, H. Nguyen, H. T. Nguyen, I. Omelyan, A. Onufriev, D. R. Roe, A. E. Roitberg, R. Salomon-Ferrer, C. Simmerling, W. Smith, J. Swails, R. C. Walker, J. Wang, R. M. Wolf, X. Wu, D. M. York and P. A. Kollman, AMBER 2014, University of California, San Francisco, 2014.

35 J. A. Maier, C. Martinez, K. Kasavajhala, L. Wickstrom, K. E. Hauser and C. Simmerling, J. Chem. Theory Comput., 2015, 11, 3696-3713.

36 W. L. Jorgensen, J. Chandrasekhar, J. D. Madura, R. W. Impey and M. L. Klein, J. Chem. Phys., 1983, 79, 926-935.

37 L. Hu and U. Ryde, J. Chem. Theory Comput., 2011, 7, 2452-2463.

38 J. Tao, J. P. Perdew, V. N. Staroverov and G. E. Scuseria, Phys. Rev. Lett., 2003, 91, 146401.

39 A. Schäfer, H. Horn and R. Ahlrichs, J. Chem. Phys., 1992, 97, 2571-2577.

40 B. H. Besler, K. M. Merz and P. A. Kollman, J. Comput. Chem., 1990, 11, 431-439.

41 U. Ryde, J. Comput.-Aided Mol. Des., 1996, 10, 153-164.

42 U. Ryde and M. H. M. Olsson, Int. J. Quantum Chem., 2001, 81, 335-347.

43 N. Reuter, A. Dejaegere, B. Maigret and M. Karplus, J. Phys. Chem. A, 2000, 104, 1720-1735.

44 L. Hu, P. Söderhjelm and U. Ryde, J. Chem. Theory Comput., 2011, 7, 761-777.

45 L. Cao and U. Ryde, Front. Chem., 2018, 6, 89.

46 F. Furche, R. Ahlrichs, C. Hättig, W. Klopper, M. Sierka and F. Weigend, Wiley Interdiscip. Rev.: Comput. Mol. Sci., 2014, 4, 91-100.

47 J. P. Perdew, K. Burke, M. Ernzerhof, B. Kieron and M. Ernzerhof, Phys. Rev. Lett., 1996, 77, 3865-3868.

48 A. D. Becke, Phys. Rev. A, 1988, 38, 3098-3100.

49 J. P. Perdew, Phys. Rev. B, 1986, 33, 8822-8824.

50 C. Lee, W. Yang and R. G. Parr, Phys. Rev. B, 1988, 37, 785-789.

51 S. Grimme, J. Comput. Chem., 2006, 27, 1787-1799.

52 J. P. Perdew, M. Ernzerhof and K. Burke, J. Chem. Phys., 1996, 105, 9982. 
53 V. N. Staroverov, G. E. Scuseria, J. Tao and J. P. Perdew, J. Chem. Phys., 2003, 119, 12129-12137.

54 A. D. Becke, J. Chem. Phys., 1993, 98, 5648-5652.

55 A. D. Becke, J. Chem. Phys., 1993, 98, 1372.

56 Y. Zhao and D. G. Truhlar, Theor. Chem. Acc., 2008, 120, 215-241.

57 Y. Zhao and D. G. Truhlar, J. Chem. Phys., 2006, 125, 194101.

58 Y. Zhao and D. G. Truhlar, J. Phys. Chem. A, 2006, 110, 13126-13130.

59 K. Eichkorn, O. Treutler, H. Öhm, M. Häser and R. Ahlrichs, Chem. Phys. Lett., 1995, 240, 283-289.

60 K. Eichkorn, F. Weigend, O. Treutler and R. Ahlrichs, Theor. Chem. Acc., 1997, 97, 119-124.

61 S. Grimme, J. Antony, S. Ehrlich and H. Krieg, J. Chem. Phys., 2010, 132, 154104.

62 S. Grimme, S. Ehrlich and L. Goerigk, J. Comput. Chem., 2011, 32, 1456-1465.

63 H. S. Yu, S. L. Li and D. G. Truhlar, J. Chem. Phys., 2016, 145, 130901.

64 T. Spatzal, J. Schlesier, E.-M. Burger, D. Sippel, L. Zhang, S. L. A. Andrade, D. C. Rees and O. Einsle, Nat. Commun., 2016, 7, 10902.

65 R. Bjornsson, F. Neese and S. DeBeer, Inorg. Chem., 2017, 56, 1470-1477.

66 P. E. M. Siegbahn, Inorg. Chem., 2018, 57, 1090-1095.

67 M. Reiher, O. Salomon and B. A. Hess, Theor. Chem. Acc., 2001, 107, 48-55.
68 K. P. Jensen and U. Ryde, J. Phys. Chem. A, 2003, 107, 7539-7545. 69 J. Li, M. Andrejic, R. A. Mata and U. Ryde, Eur. J. Inorg. Chem., 2015, 3580-3589.

70 S. Raugei, L. C. Seefeldt and B. M. Hoffman, Proc. Natl. Acad. Sci. U. S. A., 2018, 115, 10521-10530.

71 R. A. Venters, M. J. Nelson, P. A. McLean, A. E. True, M. A. Levy, B. M. Hoffman and W. H. Orme-Johnson, J. Am. Chem. Soc., 1986, 108, 3487-3498.

72 D. Lukoyanov, Z.-Y. Yang, D. R. Dean, L. C. Seefeldt and B. M. Hoffman, J. Am. Chem. Soc., 2010, 132, 2526-2527.

73 U. Ryde, R. A. Mata and S. Grimme, Dalton Trans., 2011, 40, 11176-11183.

74 J. Li, R. A. Mata and U. Ryde, J. Chem. Theory Comput., 2013, 9, 1799-1807.

75 L. Goerigk, A. Hansen, C. Bauer, S. Ehrlich, A. Najibi and S. Grimme, Phys. Chem. Chem. Phys., 2017, 19, 32184-32215.

76 M. Lundberg and P. E. M. Siegbahn, J. Comput. Chem., 2005, 26, 661-667.

77 I. Dance, Mol. Simul., 2018, 44, 568-581.

78 M. G. Delcey, K. Pierloot, Q. M. Phung, S. Vancoillie, R. Lindh and U. Ryde, Phys. Chem. Chem. Phys., 2014, 16, 7927-7938.

79 G. Dong, Q. M. Phung, S. D. Hallaert, K. Pierloot and U. Ryde, Phys. Chem. Chem. Phys., 2017, 19, 10590-10601.

80 J. N. Harvey, Annu. Rep. Prog. Chem., Sect. C: Phys. Chem., 2006, 102, 203-226.

81 S. Dohm, A. Hansen, M. Steinmetz, S. Grimme and M. P. Checinski, J. Chem. Theory Comput., 2018, 14, 2596-2608. 